\title{
SANDS9-2867C
}

\section{THE CHALLENGE OF MASSIVELY PARALLEL COMPUTING}

\author{
DAVID E. WOMBLE \\ Sandia National Laboratories, "Albuquerque, NM, USA, 87185-1110
}

\begin{abstract}
Since the mid-1980's, there have been a number of commercially available parallel computers with hundreds or thousands of processors. These machines have provided a new capability to the scientific community, and they been used successfully by scientists and engineers although with varying degrees of success. One of the reasons for the limited success is the difficulty, or perceived difficulty, in developing code for these machines. In this paper we discuss many of the issues and challenges in developing scalable hardware, system software and algorithms for machines comprising hundreds or thousands of processors.
\end{abstract}

\section{Introduction}

Massively parallel computing has been around since the mid-1980s and has been used by scientists and engineers with varying degrees of success. Presently, only a few institutions continue to use massively parallel computers. Most institutions are currently investing in machines with smaller numbers of processors or clusters of workstations and are using these machines because of their computing capacity instead of their computing capabilities.

Effective massively parallel computing has the reputation of being difficult to achieve. Whether a specific application or use of these machines is, in fact, difficult will be left up to the reader; however, it is true that there are many issues that must be specifically addressed. It is also true that these issues span the range of hardware, system software, and algorithms, and that many of these issues are beyond most users' control. Nevertheless, users must be familiar with the issues if they are to use these machines effectively ${ }^{2}$. It is the purpose of this paper to discuss the major issues affecting massively parallel computing.

We begin by defining massively parallel computing. The working definition for this paper will be the use of a sufficient number of processors that particular attention must be paid to the issues of scalability. Of course, this is application dependent. For example, in some simulations with explicit time steps, 500 processors might not be considered massively parallel. In other applications, such as those that require multidimensional FFTs, 50 proces-

"Sandia is a multiprogram laboratory operated by Sandia Corporation, a Lockheed Martin Company, for the United States Department of Energy under Contract DE-AC0494AL85000.

parco99: submitted to World Scientific on October 21, 1999 


\section{DISCLAIMER}

This report was prepared as an account of work sponsored by an agency of the United States Government. Neither the United States Government nor any agency thereof, nor any of their employees, make any warranty, express or implied, or assumes any legal liability or responsibility for the accuracy, completeness, or usefulness of any information, apparatus, product, or process disclosed, or represents that its use would not infringe privately owned rights. Reference herein to any specific commercial product, process, or service by trade name, trademark, manufacturer, or otherwise does not necessarily constitute or imply its endorsement, recommendation, or favoring by the United States Government or any agency thereof. The views and opinions of authors expressed herein do not necessarily state or reflect those of the United States Government or any agency thereof. 


\section{DISCLAIMER}

Portions of this document may be illegible in electronic image products. Images are produced from the best available original document. 
sors might be considered massively parallel. In practice, the effects of poor scalability are often seen somewhere between 50 and 100 processors. This is where slow networks, serial bottlenecks and other non-scalable constructs are almost certain to show up.

This definition reduces the challenge of efficient massively parallel computing to one of scalability. In this paper, we will consider three aspects of scalability: algorithms, hardware and system software. Each of these has an impact on overall efficiency of a simulation code as does the interaction between the three. Hardware often gets a lot of attention. Machines with tens of thousands of processors have been built, and these machines are often characterized using things like communication-to-computation ratios and bisection bandwidths.

Scalable algorithms have also been considered at length in the technical literature. Computational scientists work hard to parallelize individual applications codes and pay special attention to computational kernels like the solution of linear systems of equations, FFTs, the solution of tridiagonal systems, and matrix transposition.

The scalability of system software and tools has received somewhat less attention. Even though parallel capabilities have been added to operating systems, they often require $O\left(p^{2}\right)$ time or memory. For example, a system that requires a table entry for each page of memory on each processor requires $O\left(p^{2}\right)$ memory. Also, tools like debuggers designed for thousands of processors are almost non-existent.

In this paper, we will examine each of the three aspects of scalability in more detail. However, in light of the recent industry trend towards the use of commodity components, we first state

Fallacy \#1: A massively parallel computer is just a collection of workstations

The fact is that while workstations can be replicated and connected by reasonably fast networks, the issues of scalability discussed throughout this paper must be specifically addressed if the system is to scale to hundreds or thousands of processors. If these issues are not addressed, a cluster of a thousand workstations will be used most effectively by running a thousand small jobs simultaneously. In other words, this cluster provides computing capacity, but it does not provide a new capability to a scientist or engineer.

After the section addressing the scalability of algorithms, hardware and sytem software, we will discuss the Cplant project at Sandia National Laboratories, which is an attempt to develop a scalable cluster of workstations. 


\section{Algorithms}

Before discussing algorithms specifically, we must distinguish between two types of efficiency. The first is the traditional fixed-size parallel efficiency.

$$
E_{p}=\frac{T(n, 1)}{p T(n, p)}
$$

where $n$ is the problem size and $p$ is the number of processors on which the problem is run. This attempts to measure the effect of the parallelization, including such factors as interprocessor communications.

One of the problems with this measure of performance is that it is not consistent with how parallel machines are used. Scientists and engineers want a new capability, which usually means the ability to solve larger problems faster than would be possible on serial machines. To incorporate the effect of problem size we introduce a second type of efficiency, algorithmic efficiency, which refers to how the computational requirement scales as the number of unknowns increases or as the desired accuracy increases compared to a base problem size $n$. The algorithmic efficiency can be written as

$$
E_{a}=\frac{m T(n, p)}{T(m n, p)}
$$

where $m$ is the scaling factor for the problem size. This is, perhaps, less informative and less formal than traditional algorithm analysis. However, it does have the property that it is similar in style to the traditional parallel efficiency and that it assigns an efficiency of 1 to an algorithm that scales as $O(n)$, which is the goal of many algorithms in modeling and simulation.

Combining these efficiencies, yields

$$
E=E_{p} E_{a}=\frac{m T(n, 1) T(n, p)}{p T(n, p) T(m n, p)},
$$

which is the efficiency that scientists would actually see as they scale the problem to match machine capabilities. If $m=p$, that is if the scaling of the number of processors equals the scaling of the problem size, the efficiency becomes

$$
E=\frac{T(n, 1)}{T(p \times n, p)}
$$

which is the traditional definition of scaled efficiency where the problem size is increased in proportion to the number of processors.

An example of an algorithm that achieves good parallel efficiency, but poor algorithmic efficiency, is Jacobi iteration. An example of an algorithm 
that achieves good algorithmic efficiency, but poor parallel efficiency, is the solution of a tridiagonal linear system. If a choice must be made, an algorithm with good algorithmic efficiency is to be preferred over one with good parallel efficiency in almost all cases. This leads us to state the following.

Fallacy \#2: A massively parallel computer can make a bad algorithm good.

In other words, if the algorithm is not the preferred one for a problem of size $n$ on a serial machine, it is not likely to be the preferred one for a problem of the same size on a parallel machine.

We can also divide algorithms into two types. The first type is solvers. The term here is used broadly, and includes algorithms that are used both in serial and parallel implementations of codes. The second type is enabling algorithms. These algorithms are specific to parallel implementations, and include things like load balancing and algorithms for explicit matrix transposes. In some sense, these algorithms are purely parallel overhead, and the performance of parallel codes requires that special attention be paid to efficient, scalable implementations.

Scalability must receive special consideration when parallelizing either solver or enabling algorithms. There are two very important issues that must be addressed if a code is to be scalable.

1. Load balance. That is, the processors must have the same amount of work

2. Data locality. That is, the data needed by a processor must be in the memory of that processor.

These two issues can be combined into the single issue of data placement, although we prefer to keep them separate.

In the case of load balance, if one processor has more work than others, a serial bottleneck is created. If this extra work does not decrease in proportion to the number of processors (and this is usually the case in real applications where load imbalances are often caused by a serial component of the algorithm orimbalanced communications), scalability is severely limited. Load balancing is often considered when domain decomposition methods are used; however, it seems to receive little attention when master-slave implementations are used, and as a result, many master-slave implementations are not scalable.

The issue of data locality is not unique to parallel machines; it is also essential to good cache performance on serial machines. However, the importance of data locality is magnified on parallel machines because of increased latencies and lower bandwidths between processors (compared to local memory latencies and bandwidths). The goal of the programmer (or compiler)

parco99: submitted to World Scientific on October 21, 1999 
must be to build data structures and distribute data to processors to minimize the time spent in the overhead of interprocessor communication. The desire of programmers to ease the burden of programming parallel machine has made shared memory programming models attractive. But despite the added difficulty of explicit message passing, it has the advantage that it forces the programmer to address the issue of data locality.

Many machines are moving towards a global address space to simplify the task of parallel programming. We would like to point out here that having a global address space, by itself, does not prevent scalability. Rather it is the dependency on compilers and hardware for internode transfers and the illusion that data locality is not important that causes problems. To justify this observation, we can consider memory associated with another node as simply another layer in the memory hierarchy. It is well known that depending on compilers and hardware to restructure a code for cache performance can result in significant performance penalties, even with the relatively small latencies for a cache miss.

\section{Hardware}

Hardware receives a lot of attention. Indeed, most press releases highlight numbers of processors, theoretical peak speeds, Linpack benchmark results or some other measure of performance that focuses on the hardware capabilities. In view of this, we state

Fallacy \#3: Peak FLOPS is a good measure of a machines capabilities, and the related

Fallacy \#3a: Linpack is a good measure of a machines capabilities.

The truth is that hardware performance is an important ingredient in the high performance equation but is not by itself sufficient. Linpack plays an important role as a widely accepted benchmark and as an acceptance test, but represents accurately the performance of only a few applications.

In any parallel computer, single-processor performance and communication network performance drive overall performance. Single-processor performance, in turn, is limited by processor computing rates and by memory bus bandwidths, where the latter is the limiting factor in most modern processors. To illustrate this, we compare the DEC EV-5 and the DEC EV-6 processors. At a given clock speed, both processors have the same theoretical peak flop (floating point operations) rates. However, based on actual runs of real applications at Sandia National Laboratories, the EV-6 achieves a 2.5 to 3 times performance increase primarily through improvements in the memory bus and the processor integer unit.

parco99: submitted to World Scientific on October 21, 1999 
The performance of the interprocessor communication network is also important. Unlike computations, interprocessor communication is entirely overhead associated with parallelism. It is generally characterized in terms of latency and bandwidth, where latency is the time required to start a message transfer and bandwidth is the rate at which data can be transferred between processors. Different codes require different capabilities. Codes, like the Linpack benchmark, that are able to collect and send large messages require high bandwidths and are less sensitive to high latencies. However, codes like unstructured finite element-based simulations, tend to send smaller messages and require low latencies.

It is difficult to identify a one-size-fits-all balance between computational speeds and network latencies and bandwidths. However, in the many applications run on massively parallel computers at Sandia National Laboratories, we have found that for the current generation of processors (e.g., DEC EV-5, · Intel Pentium II), achievable latencies in the range of ten microseconds and achievable bandwidths of about $300 \mathrm{Mbytes} / \mathrm{sec}$ (approximately one byte per second for each achievable floating point operation per second) allow codes to scale to thousands of processors.

In the preceding paragraph, we skirted the issue of network topology by specifying that we use achievable bandwidths during code execution for our comparisons. However, network topology can play a crucial role in bandwidths actually achieved. For example, if a network is organized as a tree, and if the bandwidths near the trunk of the tree are no larger than those near the leaves, a code running on all processors will see significantly lower bandwidths than a code running on just two processors. This is, of course, due to the bottleneck at the trunk of the network tree.

Another important issue is the reliability of the hardware. While this does not affect a machines performance directly, it certainly affects the usability. To illustrate the point, we note that most users find it tolerable that the workstation (or PC) on their desk crashes once a month. But a machine comprising 1,000 of those same workstations would be expected to crash approximately every 45 minutes and a machine comprising 10,000 of those workstations would be expected to crash approximately every 4 minutes. This would make the machine completely unusable. Indeed, some massively parallel computers cannot even load a job in this amount of time.

In this section, we have mentioned several hardware issues affecting scalability of a computer to thousands of processors. In fact, significant progress has been, and continues to be, made by hardware vendors in all of these areas. For exampel, processor performance continues to follow the curve of Moore's law. The greater challenge to vendors of massively parallel computers

parco99: submitted to World Scientific on October 21, 1999 
is system software, which is discussed in the next section.

\section{System Software}

In this section, we discuss the impact of system software on scalability. We use the term broadly to include, not only the operating system, but also the development environment, e.g., compilers and debuggers. As with algorithms and hardware, there are significant issues that must be addressed in systems scalable to hundreds or thousands of processors.

The operating system has been and continues to be a major limitation in achieving scalablility to thousands of processors. For example, "features" that severely limit scalability include

1. Size of the operating system. Yes, memory is cheap (at present), but enough memory to load an operating system that requires tens of megabytes and that must be replicated thousands of times is still expensive. There are additional reasons to limit the size of the operating system. First, it takes significant time to boot a machine with a heavyweight operating system. Second, increased size usually means increased complexity, which means reduced stability, and it was pointed out in the previous section that the effect of reduced stability is severe in massively parallel computers.

Increased complexity also means more interactions between the OS and the users code. For example, interrupts can have the same effect as a serial bottleneck in a users code. As an extreme case, suppose a code synchronizes every 100 microseconds, that the code is perfectly balanced, that the OS generates an interrupt randomly but on average once a second, and that the interrupt lasts 10 microseconds. (In fact, Linux on an Alpha generates 1024 interupts per second.) On a two-processor machine, this results in a $0.001 \%$ reduction in scaled efficiency, because two out of every 10,000 synchronizations are delayed. (Actually, the reduction in efficiency is slightly less since there is a small chance that the two interrupts occur during the same sychronization interval.) But on a 10,000processor machine, this results in worst case scaled efficiency of $90 \%$ and an expected scaled efficiency of $94 \%$. (The number of syncrhonizations intervals that are not interrupted have a Poisson distribution with mean $10,000 / e \approx 3679$.) The fact is that many of the features that drive up the size of the OS are not necessary on the compure nodes of a massively parallel computer.

2. Support for multiple users and time sharing. Time sharing compute nodes

parco99: submitted to World Scientific on October 21, 1999 
in a massively parallel computer has the same effect (although much more severe) as OS interrupts. To illustrate this, consider a machine with 100 processors that is time shared by two users. Half of the time on each shared processor is allocated to one user who has a code loaded on all 100 processors, and half of the time is allocated to the second user who has a code loaded on 50 processors. If each job requires the same wallclock time, the total usage of the machine is only $75 \%$. A better way to use the machine is to allocate to users the number of nodes on which their code runs effectively, to guarantee them $100 \%$ of the time on those nodes, that is to space share the machine.

3. Support for virtual memory. In principle, virtual memory is a good thing, but only for workstations. The problem is that virtual memory adds a lot of overhead. Even on a workstation, a simulation that uses virtual memory can take many times longer than the same simulation running on a machine with sufficient physical memory. On a parallel computer, the situation is worse. First, swapping often appears as a serial bottleneck, in much the same way as OS interrupts described in item 1, although the effect is much worse. Second, unless there is a disk on each node, there is either network contention or disk contention as processors vie for limited disk resources.

4. Cache coherency across processors and attempts at distributed shared memory. The promise of cache coherency coupled with a global address space and a shared memory paradigm is that the user can worry about the parallelism in the computation without worrying about the parallelism in (location of) the data. The problem is that implementations of cache coherency typically scale as $O\left(p^{2}\right)$ in total memory and work. This may be fine on a very small number of processors but not on thousands of processors. Algorithms that incur $O\left(p^{2}\right)$ overhead are not considered scalable and neither should system software with the same scaling. Another problem with having this capabililty is that users will use it. But as was discussed in the algorithms section, the location of the data and the management of communications is critical in achieving scalable performance. Both of these are left to general purpose system software which is usually much less than optimal.

There are also several problems that persist in system software even though they are known to prevent scalability and have been eliminated in all successful implementations on massively parallel machines. Two examples follow.

parco99: submitted to World Scientific on October 21, 1999 
1. Linear processor loads. A job is usually started on a massively parallel computer by loading a users code on each processor on which the code is to execute. If this is done in a linear fashion, i.e., one processor at a time, the overhead is $O(p)$. However, since the same code is usually loaded on each processor, a logrithmic load is possible, which has overhead $O(\log (p))$. On a 1000-processor machine, the difference is a factor of 100: a job that should take 30 seconds to load and start would take almost an hour. This is hardly an efficient use of expensive massively parallel computer hardware.

2. Preallocated communication buffers for each processor. There are also some operating system implementations that preallocate a communication buffer corresponding to each processor from which a message is expected during the code execution (at least equal in size to the largest message expected during the execution). The problem is that for codes in which all-to-all communications are expected or possible (or if the OS cannot identify or does not allow the user to specify the communication pattern), $O\left(p^{2}\right)$ memory is required. On a 1000-processor machine, it is possible that hundreds of megabytes of memory must be reserved for communication buffers on each node.

Many of these problems arise from the mistaken view that workstation operating systems that focus on user interactions are also good for massively parallel computing. (We refer to this as a workstation mentality.) One solution is to take advantage of the fact that nodes in massively parallel computers typically serve a particular purpose and to use a partitioned model for the operating system. For example, a workstation operating system can (and should) be used on the service nodes, i.e., nodes on which users log in, compile code, and queue jobs to the compute nodes. A stripped down operating system focusing on a minimal set of services, including efficient interprocessor communications can then be used on compute nodes. A similar philosophy applies to I/O nodes, or other special-purpose nodes.

Parallel development environments and tools also suffer from a workstation mentality, although in a somewhat different fashion. The challenge in this case is to collect, process and present data from many processors in such a way that the user can quickly develop, debug or improve the performance of code for parallel computers. The problem is that most vendors are trying to simply extend current tools without sufficiently addressing the problems of scalability. Some of these challenges are the following.

1. The tools must themselves be efficient, scalable, distributed memory codes.

parco99: submitted to World Scientific on October 21, 1999 
2. The tools must be able to collect, organize and present much larger datasets to the programmer

3. There is a new set of problems that face programmers and the tools must be able to present data in such a way as to make these problems easy to identify. For example, a debugger must present data such that deadlocks due to message passing problems are obvious. This may not be difficult when programmers are explicitly programming the message passing, but it will become more difficult as compilers attempt to hide more and more of the complexities of distributed memory parallelism from the programmer.

Finally in this section, we mention compilers. Wuch has been accomplished on compilers that identify parallelism, or otherwise attempt hide or simplify the task of writing parallel code, but they are not able to produce scalable code for massively parallel computers. The problem is that while the parallelism in a simulation may be obvious at the level of the physics or the model (e.g., a domain decomposition approach), it is usually not obvious at the code level. Compilers generally consider fine-grain parallelism at the level of inner loops, whereas the physics-based parallelism is often at the level of the outer loop. As a simple example, a compiler may be able to see that a finite-difference code performs most of its calculations on one-dimensional arrays and partition the work accordingly. But it may miss the fact that the one-dimensional array comes from a three-dimensional problem and that communication may be significantly reduced if a different decomposition is used. Compilers also depend on easily understood, regular data structures to identify parallelism, but data structures are not always regular. For example, finite element simulations usually involve irregular data structures and rely heavily on indirect addressing.

Many of the tools available to programmers are effective for small numbers of processors. This represents one approach: to start with small numbers of processors and attempt to evolve the tools to large numbers of processors as techniques evolve. It is our opinion that the problems of scalability and massive parallelism, both for operating systems and tools; should be addressed up front and specifically. We will then end up with better tools and these tools will port easily to smaller numbers of processors. In other words, just as in the cases of algorithms and hardware, scalability should be addressed first.

parco99: submitted to World Scientific on October 21, 1999 


\section{Cplant}

Tightly-integrated, custom designed massively parallel machines have continued to increase in cost until very few institutions or industries are able to afford them or willing to buy them. As a result, the recent trend is to assemble massively parallel machines as much as possible from commodity (workstation and network) components. The trap in this approach is to fall into the workstation mentality, that is that massively parallel computers can be designed and used just like workstations. The result of this thinking is a machine with a large capacity, but one that does not provide a new capability to scientists or engineers. (Of course, to be fair, capacity may be exactly what is needed in many cases.)

Sandia National Laboratories has begun a project to design, build and put into production a scalable machine of commodity components that in fact provides a new capability ${ }^{1}$. The machine is called Cplant. This machine currently consists of over 1,000 DEC EV-5 and EV- 6 based workstations with a Myrinet interprocessor network and a version of the Linux operating system on each node. The largest single "piece" of Cplant is currently 604 nodes.

Scalability is being addressed from the start.

1. System designers are working to provide as low latency and as much of the myrinet bandwidth to applications as possible. This has involved developing extensions to Linux focused on message passing. Furthermore, the network has been designed so as not to introduce communication bottlenecks between switches.

2. Designers have addressed the reliability problem both through good hardware design (e.g., proper cooling and power supplies), through strong diagnostics, and by including a diagnostic network that enables management of individual nodes.

3. Designers are building a range of tools, including debuggers and resource management (e.g., queuing) that specifically address the massively parallel computing environment.

4. Designers have used a partitioned approach. Nodes have specific functions (e.g., computation, service, I/O or management), which enables the software on each node to do one thing reliably and well.

The Cplant focus has been on the hardware and system software aspects, and in this they have been successful. Codes that scale well to thousands of processors on machines like Sandia's Teraflops compter currently scale to 
hundreds of processors on Cplant. It is expected that these codes will scale to thousands of processors with a scheduled doubling of the bandwidth of the communication network and with continued improvements in such areas as operating system design and I/O.

\section{Conclusions}

The primary conclusion of this paper is that scalability takes extra effort and investment in algorithms, hardware and system software. In the case of algorithms, this responsibilty falls to the user or code developer, although parallel libraries and other common software can greatly reduce the work. The primary issue for the code developer is data placement (data locality and load balance). Also, parallelism is found primarily in the physics of the problem, not in the code, and the most effective code is the product of a developer who understands the problem.

The scalability of hardware and system software is most often the responsibility of the vendor. For hardware, the primary issue is providing communication between processors that matches the computational capabilities of today's processors. For system software, the challenge is to provide a robust operating system with scalable algorithms and without serial bottlenecks that and tools that collect, process and present the large amounts of data generated by massively parallel computers. For both hardware and system software, the issue of reliability must be addressed.

In each of these areas, we have found a "workstation mentality," that is, the desire to ignore the challenge of scalability and to design and use a massively parallel computer in exactly the same fashion as a workstation. This approach almost always leads to failure. Scalability must be designed in from the beginning, and the reward for doing so is a new capability for the scientist and engineer.

\section{Acknowledgement}

The author would like to thank the many staff members at Sandia National Laboratories who have contributed their experience to their paper through discussions of scalability. These staff members include Mike Heroux, Bruce Hendrickson, Steve Plimpton, Jim Tomkins, and Rolf Riesen.

\section{References}

1. R. Riesen, http://www.cs.sandia.gov/cplant, (1999). 
2. D. E. Womble et al, Paralel Computing 26, to appear (2000). 